\title{
The Use of Water To Reduce Physiological Infra- Cardiac Tracer Uptake in Stress Myocardial Perfusion Imaging Using Single Photon Emission Computed Tomography: First Experience in Indonesia
}

Nora Anggun Prasetyo ( $\nabla$ noraprasetyo2004@gmail.com )

Padjadjaran University

\section{Erwin Affandi Soeriadi}

Dr. Hasan Sadikin General Hospital

Hendra Budiawan

Dr. Hasan Sadikin General Hospital

Achmad Hussein Sundawa Kartamihardja

Padjadjaran University

\section{Research Article}

Keywords: Milk, SPECT, stress Myocardial Perfusion Imaging procedure, water.

Posted Date: September 7th, 2021

DOI: https://doi.org/10.21203/rs.3.rs-752762/v1

License: (9) This work is licensed under a Creative Commons Attribution 4.0 International License. Read Full License 


\section{Abstract}

\section{Background}

Coronary artery disease (CAD) remains an important cause of morbidity and the main global cause of death. Myocardial perfusion imaging (MPI) used in diagnosing and evaluating in myocardial ischemia has been shown to be more cost effective and sensitive than other modalities. Milk is usually used to reduce the physiological uptake by infra-cardiac organs. However, some of our patients were lactose intolerant that can cause diarrhea and abdominal discomfort.

The aim of this study was to evaluate the use of water, instead of milk, to reduce the physiological infracardiac tracer uptake.

\section{Methods}

Sixty patients were referred to undergo MPI procedure between October 2020 to December 2020. They were divided into 2 groups, first group received $500 \mathrm{~mL}$ of milk and the second group received $330 \mathrm{~mL}$ of water after MIBI injection. Stress procedure was performed using treadmill with modified Bruce protocol. Image acquisitions were performed 30 minutes after MIBI injection. A semi-quantitative assessment was made by evaluating the myocardial activity and the infra-cardiac tracer uptake. Qualitative assessment was made by determining whether the infra-cardiac tracer uptake interferes the MPI interpretation.

\section{Results}

Patients consist of 30 males and 30 females. The age of the patients was between $29-80$ years old (mean: 53.3). The semi-quantitative and qualitative images were done in both groups. The results showed that, there were no physiological infra-cardiac tracer uptake in both groups, which caused interference.

\section{Conclusions}

Water is able to reduce physiological infra-cardiac tracer uptake and it can be used in patients with lactose intolerant.

\section{Trial registration}

Not applicable.

\section{Background}

Coronary artery disease (CAD) remains an important cause of morbidity and the main global cause of death. According to the World Health Organization (WHO) estimation, more than 9 million deaths in 2016 due to $C A D^{[1]}$. 
Myocardial perfusion imaging (MPI) used in diagnosing and evaluating in myocardial ischemia has been shown to be more cost effective and sensitive than other modalities ${ }^{[2-4]}$.

High infra-cardiac (liver, bowel or gastric) tracer uptake can interfere the interpretation of MPI, especially the inferior wall and could cause false interpretation ${ }^{[5,6]}$, Milk is usually used to reduce the physiological uptake by infra-cardiac organs. Some of our patients were lactose intolerant that can cause diarrhea and abdominal discomfort. Indonesian population have high prevalence of lactose malabsorption and intolerance ${ }^{[7-9]}$. Study conducted by Peace and Lloyd showed that there were no differences were found in the quantitative results between milk and water on MPI procedure ${ }^{[10]}$.

The aim of this study was to evaluate the use of water, instead of milk, to reduce the physiological infracardiac tracer uptake.

\section{Methods}

The study population was patients who referred to our department for MPI procedure between October 2020 to December 2020. Informed consent was obtained from each subject before conducted the procedure. Subjects who had medically essential fluid restriction, consume anti-motility drug (loperamide, codeine, morphine) or pro-motility drug (metoclopramide, domperidone, erythromycin, antiemetic, antihistamine, antidepressant), pregnant, and lactating were excluded from this study. All subjects were asked to fast for at least 4 hours prior MPI procedure.

Subjects divided into 2 groups, first group received $500 \mathrm{~mL}$ of milk (this procedure is the same as the MPI procedure that we are used to in our department) and the second group received $330 \mathrm{~mL}$ of water following MIBI injection.

Stress procedure was performed using treadmill with modified Bruce protocol. Image acquisitions were taken 30 minutes after MIBI injection in supine position.

A semi-quantitative assessment was made by evaluating radioactivity in myocardium and infra-cardiac tissues. Score 0 , if there is no infra-cardiac radioactivity or lower than myocardial radioactivity; score 1, if infra-cardiac radioactivity equal to or higher than myocardial radioactivity. A qualitative assessment was made by determining whether the infra-cardiac activity interferes the MPI interpretation. The assessments were made by three nuclear medicine specialists with more than 3 years experiences in nuclear cardiology. Images were presented randomly to interpreters who were blinded to clinical history of the subjects. The agreement reached when there is a consensus between minimal two nuclear medicine specialist.

All procedures performed in this studies that involving human participants were approved and carried out in accordance with the ethical standards of the Health Research Ethics Committee of Dr. Hasan Sadikin General Hospital Bandung (West Java - Indonesia) and/or national research committee. Ethical Approval 
Number: LB.02.01/X.6.5/311/2020. Written informed consent was obtained from all the patients those were enrolled in the study.

\section{Results}

A total of 60 subjects (30 males and 30 females) with age of between 29-80year old (mean age: $53.3 y e a r$ old) were included in this study. All subjects achieved at least $85 \%$ of maximal heart rate in stress test MPI procedure. Subject characteristics were shown in Table 1.

Table 1

Subjects characteristics and results

\begin{tabular}{|lll|}
\hline Characteristics & Water $(\mathbf{n = 3 0})$ & Milk $(\mathbf{n = 3 0})$ \\
\hline Male & 16 & 14 \\
\hline Female & 14 & 16 \\
\hline Age (years old) & $28-80$ (mean: 48.7) & $39-71$ (mean: 58.3$)$ \\
\hline
\end{tabular}

Both semi-quantitative and qualitative assessments, were done in both groups. The result showed, there was no physiological infra-cardiac organ tracer uptake in both groups, which caused interference in both groups as shown in Fig. 1, Fig. 2, Table 2, and Table 3.

Table 2

Intensity of infra-cardiac and myocardial tracer uptake

\begin{tabular}{|lll|}
\hline & $(0) *$ & $(1) * *$ \\
\hline Milk & $30(100 \%)$ & 0 \\
\hline Water & $30(100 \%)$ & 0 \\
\hline
\end{tabular}

*) There is no infra-cardiac radioactivity or lower activity than myocardial radioactivity.

**) Infra-cardiac radioactivity equal to or higher than myocardial radioactivity. 
Table 3

Interference of infra-cardiac tracer

uptake on interpretation

\begin{tabular}{|lll|}
\hline & Interfere & Not Interfere \\
\hline Milk & 0 & $30(100 \%)$ \\
\hline Water & 0 & $30(100 \%)$ \\
\hline
\end{tabular}

\section{Discussion}

CAD is the most common type of cardiovascular disease and the main cause of mortality and morbidity. Radionuclide myocardial perfusion imaging can be used to determine the presence of CAD, give risk stratification and to guide management of patients with known disease ${ }^{[11,12]}$. Single Photon Emission Computed Tomography (SPECT) MPI using ${ }^{99 \mathrm{~m} T c-S e s t a m i b i ~(M I B I)}$ is cost effective than any other diagnostic modalities and more accurate than exercise ECG in detecting myocardial ischemia. It can be used to evaluate myocardial viability before revascularization and following-up after revascularization such as coronary artery bypass graft or angioplasty ${ }^{[2-4]}$. MPI was confirmed to be highly effective in the diagnosis of CAD, with sensitivity of $85-95 \%$, specificity of $75 \%$, and a normalcy rate of $88 \%{ }^{[2]}$.

Stress images in SPECT may help determine the degree of inducible ischemia or viable myocardium. This viable myocardium will get advantage if the patient undergo revascularization ${ }^{[13]}$.

According to European Association of Nuclear Medicine (EANM) guidelines, the exercise stress test is preferred than pharmacological stress. Exercise stress allows evaluation of imbalance between oxygen supply and demand due to impaired flow reserve. It can identify the ischemic area related to heart workload. Images should be acquired 30-60 minutes after injection to allow adequate hepatobiliary clearance ${ }^{[14]}$. Meanwhile, according to SNMMI guideline, stress imaging should be taken between 15-60 minutes post-tracer injection ${ }^{[15]}$. The acquisition time we used in this study was 30 minutes after tracer injection.

High liver, bowel or gastric tracer uptake can interfere interpretation of $\mathrm{MPI}$, especially the inferior wall and lead to false positive interpretation in normal MPI. Although this phenomenon can be reduced using iterative reconstruction and attenuation correction, a high tracer uptake around the heart may disturb the myocardial tracer uptake evaluation ${ }^{[5,6]}$. Some centers ask the patients to consume fatty meal between injection and imaging time to increase clearance of tracer from the liver and gallbladder. In patient with retrograde passage of tracer from duodenum to stomach or the tracer reach the transverse colon, this maneuver may be counterproductive ${ }^{[14]}$.

In our department, the standard stress SPECT MPI protocol is to drink $500 \mathrm{~mL}$ milk after MIBI injection 30 minutes before acquisition. However, some patients complain of diarrhea and abdominal discomfort 
after drinking the milk. Fluid intake is also believed can be used to remove intestinal activity from the subdiaphragmatic area ${ }^{[14]}$.

In this study, we asked subject to drink $500 \mathrm{~mL}$ milk for the first group, and $330 \mathrm{~mL}$ of water for the second group following MIBI injection. We exclude subject who have history of fluid restriction, consume pro-motility drug such as metoclopramide, because it can increase the gastrointestinal tracer uptake activity.

Our study showed that water was as good as milk in reducing the infra-cardiac tissue tracer uptake. This result was similar to a prospective study conducted by Hoffman et. al. Their study demonstrated that there was no difference between milk and water on MPI interpretation ${ }^{[16]}$. Several previous studies have been conducted to find a substitute for milk in MPI procedure. Hurwitz et. al. using milkshake and found that decreased in infra-cardiac tracer uptake was due to volume of stomach ${ }^{[17]}$. Boz et. al. gave normal meal and drink 200-300 mL water 10-15 minutes before imaging. They hypothesized that filled stomach will push the intestinal caudally and set apart intestinal from myocardium ${ }^{[18]}$. Hara et. al. used $100 \mathrm{~mL}$ of soda water in their study, and their results showed that the mean inferior wall to abdomen count ratio was significantly higher in the soda water group than control ${ }^{[19]}$. Thomas et. al. also found the same result on their study about the use of carbonated water in reduction of adjacent gastric activity [20].

All of those previous studies showed that soda water, milk, and water could be used to minimize the infracardiac tracer uptake which can interfere interpretation of SPECT MPI.

Our qualitative assessments showed that both water and milk reduced physiological infra-cardiac organ tracer uptake and no difference in semi-quantitative assessment or in interpretation of the MPI result. Therefore, for patients with history of lactose intolerant, they can drink water instead of milk to get high quality MPI result with more convenience.

\section{Conclusion}

Drink water is an alternative approach to reduce physiological infra-cardiac organ tracer uptake in stress SPECT MPI.

\section{List Of Abbreviations}

1. CAD: Coronary Artery Disease

2. EANM: European Association of Nuclear Medicine

3. MIBI: ${ }^{99 m}$ Tc-Sestamibi

4. MPI: Myocardial Perfusion Imaging

5. SPECT: Single Photon Emission Computed Tomography 
6. WHO: World Health Organization

\section{Declarations}

\section{Ethics approval and consent to participate}

All procedures performed in this study involving human participants were in accordance with the ethical standards of Dr. Hasan Sadikin General Hospital Bandung and had been approved by the Health Research Ethics Committee of Dr. Hasan Sadikin General Hospital Bandung (West Java - Indonesia) (Ethical Approval Number: LB.02.01/X.6.5/311/2020). Written informed consent was obtained from all the patients those were enrolled in the study.

\section{Consent for publication}

Consent for publication was included in written informed consent that was obtained from all the patients those were enrolled in the study.

\section{Availability of data and materials}

The datasets used and/or analysed during the current study are available from the corresponding author on reasonable request.

\section{Competing interests}

The authors declare that they have no competing interests.

\section{Funding}

There was no source of funding for this study.

\section{Authors' contributions}

NAP made the concept of this study, and design this study. She also did the literature search, performed the study, did the data acquisition, analysed the datasets, and analysed the statistic too. She prepared the manuscript, edited it and the reviewed it.

EAS, HB, and AHSK helped in generating the concept of this study, and designing this study. They also helped in editing and reviewing the manuscript.

AHSK acted as the guarantor of this study.

\section{Acknowledgements}

Not applicable. 


\section{References}

1. Nowbar, A. N., Gitto, M., Howard, J. P., Francis, D. P. \& Al-Lamee, R. Mortality From Ischemic Heart Disease. Circ Cardiovasc Qual Outcomes 12, e005375, doi:10.1161/CIRCOUTCOMES.118.005375 (2019).

2. Loong, C. Y. \& Anagnostopoulos, C. Diagnosis of coronary artery disease by radionuclide myocardial perfusion imaging. Heart 90 Suppl 5, v2-9, doi:10.1136/hrt.2003.013581 (2004).

3. Zellweger, M. J. et al. When to stress patients after coronary artery bypass surgery? Risk stratification in patients early and late post-CABG using stress myocardial perfusion SPECT: implications of appropriate clinical strategies. J Am Coll Cardio/37, 144-152, doi:10.1016/s0735-1097(00)01104-9 (2001).

4. Zaret, B. L., Strauss, H. W., Martin, N. D., Wells, H. P., Jr. \& Flamm, M. D., Jr. Noninvasive regional myocardial perfusion with radioactive potassium. Study of patients at rest, with exercise and during angina pectoris. N Engl J Med 288, 809-812, doi:10.1056/NEJM197304192881602 (1973).

5. Germano, G., Chua, T., Kiat, H., Areeda, J. S. \& Berman, D. S. A quantitative phantom analysis of artifacts due to hepatic activity in technetium-99m myocardial perfusion SPECT studies. J Nucl Med 35, 356-359 (1994).

6. Hambye, A. S., Delsarte, P. \& Vervaet, A. M. Influence of the different biokinetics of sestamibi and tetrofosmin on the interpretation of myocardial perfusion imaging in daily practice. Nucl Med Commun 28, 383-390, doi:10.1097/MNM.0b013e3280e40d85 (2007).

7. Lukito, W., Malik, S. G., Surono, I. S. \& Wahlqvist, M. L. From 'lactose intolerance' to 'lactose nutrition'. Asia Pac J Clin Nutr 24 Suppl 1, S1-8, doi:10.6133/apjcn.2015.24.s1.01 (2015).

8. Swagerty, D. L., Jr., Walling, A. D. \& Klein, R. M. Lactose intolerance. Am Fam Physician 65, 18451850 (2002).

9. Hegar, B. \& Widodo, A. Lactose intolerance in Indonesian children. Asia Pac J Clin Nutr 24 Suppl 1, S31-40, doi:10.6133/apjcn.2015.24.s1.06 (2015).

10. Peace, R. A. \& Lloyd, J. J. The effect of imaging time, radiopharmaceutical, full fat milk and water on interfering extra-cardiac activity in myocardial perfusion single photon emission computed tomography. Nucl Med Commun 26, 17-24, doi: 10.1097/00006231-200501000-00004 (2005).

11. Khan, D. T. Cardiovascular Diseas. Preprint at https://www.who.int/health-topics/cardiovasculardiseases\#tab=tab_1 (2020).

12. Kostkiewicz, M. Myocardial Perfusion Imaging in Coronary Artery Disease. The Czech Society of Cardiology 57, 446-452, doi:10.1016/j.crvasa.2015.09.010 (2015).

13. Hachamovitch, R., Hayes, S. W., Friedman, J. D., Cohen, I. \& Berman, D. S. Comparison of the shortterm survival benefit associated with revascularization compared with medical therapy in patients with no prior coronary artery disease undergoing stress myocardial perfusion single photon emission computed tomography. Circulation 107, 2900-2907, doi:10.1161/01.CIR.0000072790.23090.41 (2003). 
14. Hesse, B. et al. EANM/ESC procedural guidelines for myocardial perfusion imaging in nuclear cardiology. Eur J Nucl Med Mol Imaging 32, 855-897, doi:10.1007/s00259-005-1779-y (2005).

15. Dorbala, S. et al. SNMMI/ASNC/SCCT guideline for cardiac SPECT/CT and PET/CT 1.0. J Nucl Med 54, 1485-1507, doi:10.2967/jnumed.112.105155 (2013).

16. Hofman, M., McKay, J. \& Nandurkar, D. Efficacy of milk versus water to reduce interfering infracardiac activity in 99mTc-sestamibi myocardial perfusion scintigraphy. Nucl Med Commun 27, 837842, doi:10.1097/01.mnm.0000237989.60196.71 (2006).

17. Hurwitz, G. A., Clark, E. M., Slomka, P. J. \& Siddiq, S. K. Investigation of measures to reduce interfering abdominal activity on rest myocardial images with Tc-99m sestamibi. Clin Nucl Med 18, 735-741, doi:10.1097/00003072-199309000-00001 (1993).

18. Boz, A. \& Karayalcin, B. Which is better for inferior wall evaluation: a full or empty stomach? J Nucl Med 37, 1916-1917 (1996).

19. Hara, M. et al. Reduction of infracardiac intestinal activity by a small amount of soda water in technetium-99m tetrofosmin myocardial perfusion scintigraphy with adenosine stress. J Nucl Cardiol 15, 241-245, doi:10.1016/j.nuclcard.2007.09.029 (2008).

20. Thomas, D. M. et al. Use of carbonated water in reduction of adjacent gastric activity in 456 consecutive technetium-99m myocardial perfusion imaging studies. Ther Adv Cardiovasc Dis 9 , 366-374, doi:10.1177/1753944715593905 (2015).

\section{Figures}
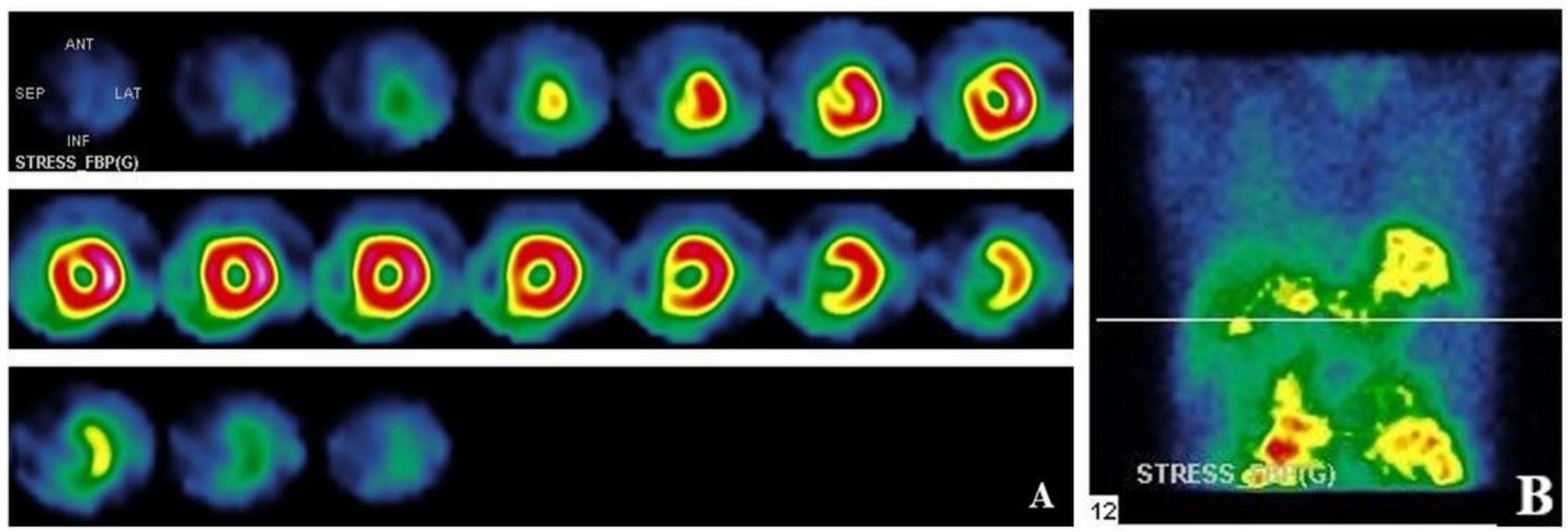

\section{Figure 1}

Male 66 years old who received milk after MIBI injection (A): in short axis, there is no interference from infra-cardiac tracer uptake at inferior wall (B): in cinematic display, there was increased clearance of the tracer from liver and gall-bladder, resulting the decrease of infra-cardiac tracer uptake. 

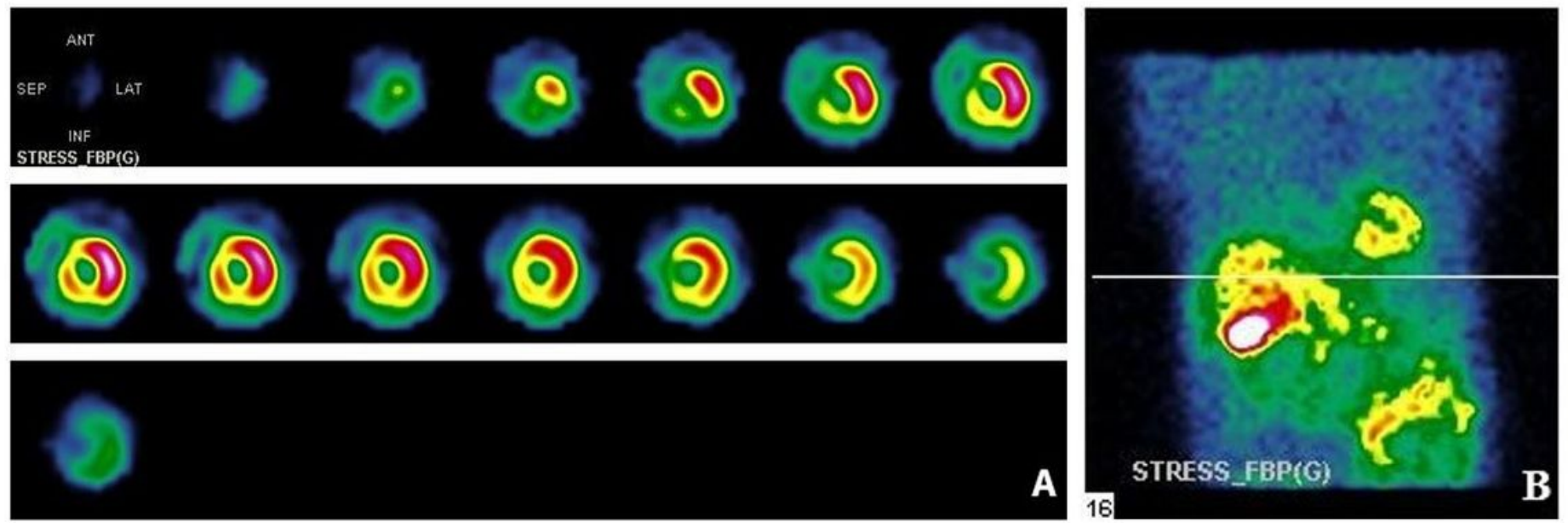

\section{Figure 2}

Male 70 years old who received water after MIBI injection (A): in short axis, there is no interference from infra-cardiac tracer uptake at inferior wall (B): in cinematic display, showed that water filled stomach and push the intestinal caudally and set apart intestinal from myocardium. 